\title{
Riesgo por inundaciones en asentamientos precarios del periurbano. Morelia, una ciudad media mexicana. ¿El desastre nace o se hace? 1
}

\author{
Juan Hernández² y Antonio Vieyra ${ }^{3}$
}

\begin{abstract}
RESUMEN
Durante las últimas décadas las ciudades en riesgo se han incrementado considerablemente, en especial sobre países en desarrollo, estimulado por el crecimiento demográfico y la constante reconfiguración urbana. Estos parámetros se manifiestan en la expansión urbana, conformando sectores periurbanos marginales y precarios que al ser impactados por eventos de inundaciones magnifican sus condiciones de vulnerabilidad. La ciudad de Morelia en los últimos diez años ha manifestado la recurrencia de esta problemática, reflejándose en las características sociorresidenciales periféricas, debido al incremento de población en situación precaria. Por lo tanto, las consecuencias de las inundaciones suelen ir acompañadas de diversas decisiones y condiciones humanas, de tal forma, este trabajo parte de la correlación de variables físicas y sociales, así como del uso de diversos métodos y herramientas para obtener los parámetros necesarios en el reconocimiento de áreas precarias y su relación con el riesgo de desastre por inundaciones.
\end{abstract}

Palabras clave: Precariedad, inundaciones, periurbano, riesgo, vulnerabilidad.

\begin{abstract}
During the past decades, cities at risk have considerably increased in number, particularly in developing countries, stimulated by demographic growth and constant urban remodeling. Such parameters are expressed in urban expansion, creating marginal and precarious peri-urban sectors that when impacted by flooding events magnify their vulnerable condition. During the past ten years the city of Morelia has repeatedly shown such problematic due to the increase in precarious populations, which is in turn, is a reflection of the peripheral socio-residential characteristics. Therefore, the consequences of flooding tend to be accompanied by different decisions and human conditions. The present work branches off from the correlation of physical and social variables, as well as from the use of a number of methods and tools in order to obtain the needed parameters for the recognition of precarious areas and their relationship with risk of disaster due to flooding.
\end{abstract}

Key words: Precariousness, flooding, peri-urban, risk, vulnerability.

\footnotetext{
1 Este trabajo es resultado del proyecto de investigación "Urbanización Deterioro Ambiental y Precariedad Urbana en Morelia, Michoacán", que contó con financiamiento PAPIIT-UNAM (IN303309-2) y se llevó a cabo en el Centro de Investigaciones en Geografía Ambiental de la Universidad Nacional Autónoma de México. Artículo recibido el 1 de junio de 2010 y aceptado el 18 de agosto de 2010.
}

2 Programa de Posgrado en Geografía (Manejo Integral del Paisaje), Universidad Nacional Autónoma de México (México). E-mail: jaher@pmip.unam.mx

3 Centro de Investigaciones en Geografía Ambiental, Universidad Nacional Autónoma de México (México). E-mail: avieyra@ciga.unam.mx 
Los desastres urbanos asociados a fenómenos naturales han tenido repercusiones diferentes a través de la historia, sin embargo, durante la última década los impactos de amenazas naturales han causado innumerables pérdidas humanas, materiales y económicas (Pelling, 2005), principalmente en ciudades pequeñas y medianas latinoamericanas, debido a las condiciones humanas y los procesos acelerados de consolidación de suelo que se están Ilevando a cabo.

Bajo este proceso, cabe destacar las problemáticas ambientales y sociales en las periferias, donde se observa la constante incorporación de tierras sin una vocación urbana, invadiendo suelo productivo, transformando ecosistemas y cambiando la habitabilidad de la población local. De la misma forma, estos espacios comúnmente son ocupados por población de bajos recursos económicos, que dadas sus necesidades de vivienda, se asientan sobre suelos inseguros y factibles a sufrir un desastre (Aguilar y Escamilla, 2009; Zulaica y Celemín, 2008; Bull-Kamanga et al., 2003).

Ante tales circunstancias, en el contexto multifactorial y multicausal del desastre es fundamental remitirse al concepto de riesgo, debido a los elementos que lo integran y favorecen su formación. El riesgo se encuentra intrínsecamente ligado a las actividades humanas, determinado por un agente perturbador (amenaza) que ocasiona algún tipo de daño a un sistema afectable (vulnerabilidad). Entre las amenazas que han causado los mayores estragos en las áreas urbanas, destacan las de origen hidrometeorológico, debido a la intensidad y magnitud con que estas se presentan. Cabe destacar que las afectaciones repercuten en mayor proporción sobre la población vulnerable, que acorde a los factores físicos, sociales, económicos y ambientales incrementan la susceptibilidad ante los impactos (Cutter et al., 2003; Cannon et al., 2004).

En este sentido, las tormentas severas vinculadas a la reconfiguración urbana, condiciones y situaciones humanas han incrementado la formación de inundaciones, siendo los asentamientos precarios los que comúnmente son más afectados, debido a la inestabilidad, transitoriedad, inseguridad, carencias y fragilidad en que se encuentran las viviendas, destacando como factores principales la materialidad, ubicación, servicios básicos y tenencia de la tierra. Así también intervienen directamente aspectos de ingresos, educación y servicios de salud, lo que repercute en las formas de respuesta, resistencia y recuperación por parte de la población damnificada (García, 2008; Lavell, 2002; Cardona, 2003).

En relación a lo establecido, la fase avanzada de transición y fragmentación urbana que presenta actualmente México es similar a la realidad latinoamericana, donde se aprecia la proliferación y crecimiento de ciudades medias y pequeñas, caracterizadas por estructuras centralizadas, concentración de economías regionales, cultura, inversión y poder político (Azócar et al., 2008). De la misma forma la expansión periférica es constante y se caracteriza por asentamientos precarios, donde su principal resguardo es la vivienda autoconstruida con materiales naturales y ligeros, carencia de los servicios más básicos e irregularidad en la tenencia de la tierra (Aguilar \& Vieyra, 2008; Cortés et al., 2003); esta situación formaliza enclaves precarios en riesgo de desastre por inundaciones, siendo el caso específico, para este trabajo, la ciudad de Morelia.

\section{Antecedentes}

La ciudad de Morelia desde su fundación (principios del siglo XVI) ha presenciado diferentes episodios de inundaciones, sin embargo, en los últimos diez años la problemática se ha magnificado a tal grado que las comunidades han sido incapaces de sufragar su afectación. Estas circunstancias se asocian a la rápida conformación de asentamientos humanos sobre terrenos irregulares, mismos que han sido producto de invasiones, negociaciones clandestinas o procesos de corruptela. Bajo este escenario, la periferia de la ciudad presenta una dinámica y presión más fuerte que la ciudad central, consolidando espacios que hasta hace unos años eran dedicados a las actividades del sector primario (agricultura, ganadería y forestal).

Por lo tanto, el proceso de urbanización se extendió hacia tierras de cultivo y antiguas haciendas que bordeaban la ciudad, lo que favoreció la negociación y especulación del 
suelo, en especial, sobre la periferia urbana (Ávila, 2007). En base a ello, diversos asentamientos se situaron sobre los límites naturales de los principales ríos (río Grande y río Chiquito), antiguas ciénegas (desecadas debido a la falta de saneamiento, proliferación de enfermedades e inundaciones) y depresiones naturales de captación de agua. Cabe destacar que una gran mayoría de estos asentamientos periféricos conformaron enclaves de pobreza y precariedad, caracterizados por la deficiente calidad de la vivienda, carencia de servicios (drenaje, agua potable y luz eléctrica) e irregularidad en la tenencia del suelo.

Con base en lo mencionado, el río Grande y el río Chiquito que atraviesan la ciudad de Morelia y sus respectivos afluentes (entre ellos el arroyo de Tierras, presente en los temporales de lluvias) son considerados peligrosos debido a sus constantes desbordes (Silva y Arreygue, 2005; Arreygue et al., 2005). Sin embargo, en los últimos años la incidencia de inundaciones en la periferia de la ciudad ha cobrado innumerables pérdidas materiales y económicas, lo que Ileva a suponer los cambios estructurales a raíz de la extensión ilimitada de superficie urbana y la proliferación de asentamientos, favoreciendo la formación de nuevas zonas de inundaciones y agudizando la problemática en aquellas que constantemente han sufrido los impactos.

Dentro de las inundaciones que han afectado seriamente a la ciudad de Morelia destaca la ocurrida en el 2002, como consecuencia de tormentas severas, dejando pérdidas de aproximadamente 4 millones de dólares. Sin embargo, el evento de inundación registrado en septiembre de 2003, producto también de tormentas rigurosas y el desasolve de la presa Cointzio (cuenca arriba), ha sido considerado como el segundo en mayores proporciones. Para el año de 2005 se presentaron las mayores inundaciones registradas, dañando viviendas e infraestructura, suspensión de servicios, así como disposición de albergues para los pobladores de distintas colonias, en el primer plano y periferia de la ciudad.

Los antecedentes obtenidos muestran que la mala localización de asentamientos, el aumento del área urbana, la calidad de la vivienda, las precipitaciones de gran intensi- dad y magnitud, así como las condiciones de la población, han incrementado el riesgo de desastre por inundaciones en la periferia de la ciudad en los últimos años. Por lo anterior, este trabajo tiene por objetivo la correlación de las áreas urbanas precarias y las zonas de inundaciones, para definir las áreas que potencialmente se encuentran en condición de riesgo sobre el periurbano de la ciudad de Morelia. Para ello, el proceso metodológico parte de argumentos multivariables y métodos hidrológicos, los cuales están desarrollados a partir de variables físicas y sociales, mismos que serán correlacionados para obtener las zonas precarias en riesgo de desastre por inundaciones.

\section{Descripción del área de estudio}

La ciudad de Morelia se localiza en la región centro-norte del estado de $\mathrm{Mi}$ choacán, en el Ilamado antiguo "Valle de Guayangareo" ${ }^{4}$, entre las coordenadas $19^{\circ}$ $38^{\prime}$ y $19^{\circ} 50^{\prime} \mathrm{N}$ y los $101^{\circ} 06^{\prime}$ y $101^{\circ} 18^{\prime}$ W, con una altitud de 1.920 m.s.n.m. y una superficie de 9.804.4 hectáreas (Figura № 1 ).

La zona de estudio forma parte del Cinturón Volcánico Mexicano (CVM), bordeada por los volcanes de Atécuaro, Punhuato, El Quinceo, El Águila y Las Tetillas. Las principales corrientes superficiales son el río Grande (transversal a la ciudad oeste a este) y el río Chiquito (sur a norte), así también en los temporales de lluvias se presenta el afluente denominado arroyo de Tierras (Silva y Arreygue, 2005). El clima se caracteriza por ser templado subhúmedo con una temperatura que oscila entre los 16,2 y $18,7^{\circ} \mathrm{C}$. Por su parte, la precipitación pluvial se presenta principalmente durante el temporal de lluvias (mayo a octubre), con una media anual de $780 \mathrm{~mm}$.

4 Guayangareo fue como se le bautizó a esta región en el año de 1522, antes de los nombres de Ciudad de Mechuacán (1541), Valladolid (1545) y la actual Morelia (1828), este último nombre deriva del apellido de José María Morelos y Pavón, destacado personaje en la lucha de independencia (Secretaría de Desarrollo Social, 2007). 
Figura $N^{0} 1$

Localización de la ciudad de Morelia

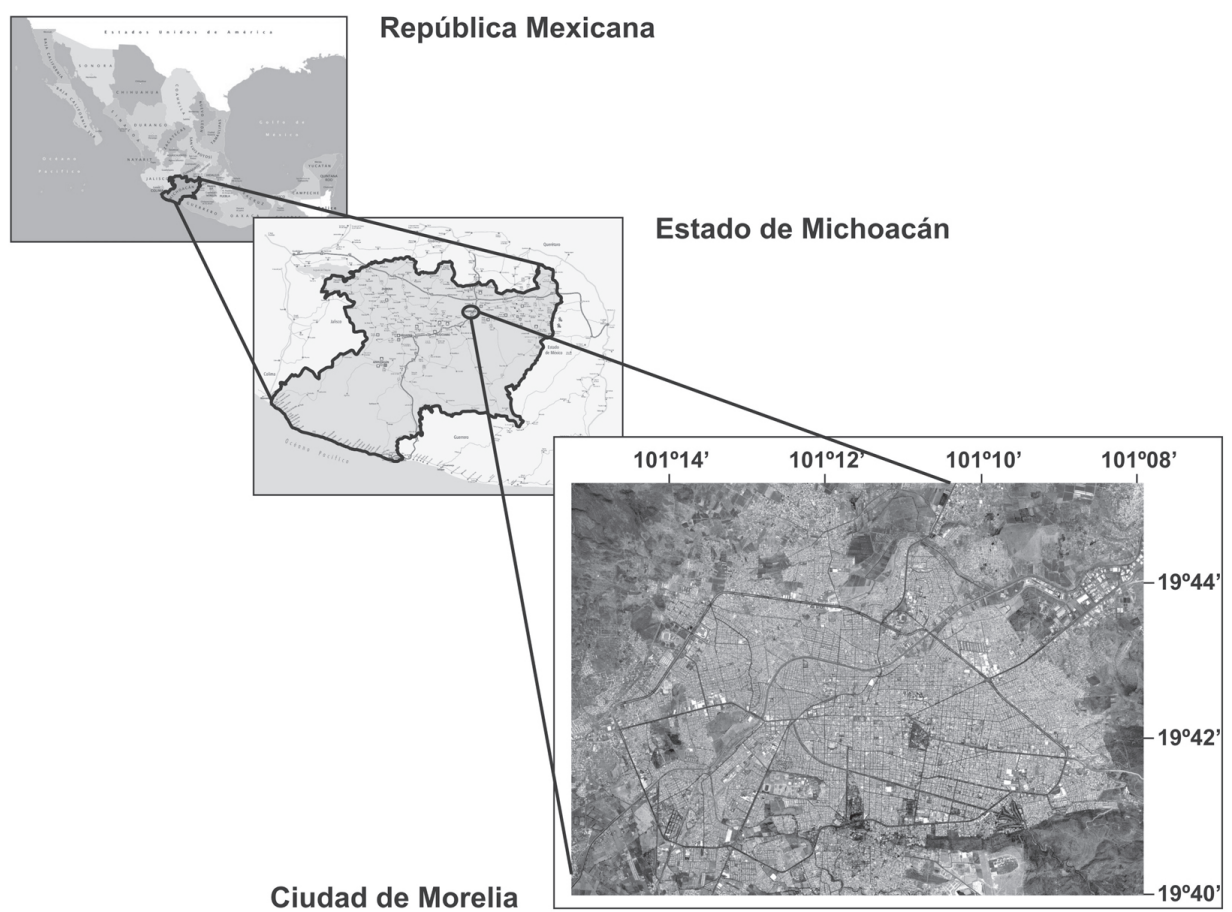

Fuente: Elaboración propia.

\section{Metodología}

En base a la multidimensionalidad de la problemática, la metodología parte del proceso de elementos cualitativos y cuantitativos, así como la adecuación de diferentes métodos para su aplicación. El trabajo consta de tres etapas principales, en la primera de ellas se determinaron las zonas de inundaciones naturales $u$ originales a través de parámetros de precipitación y morfométricos (precipitaciónescorrentía), en otras palabras, no se utilizó red hidráulica o estaciones hidrométricas para su estimación. Por lo tanto, para comprender el factor correspondiente al comportamiento de la lluvia se utilizó el Método de Análisis Espectral, mientras que el segundo se apoyó en los resultados de la precipitación y se definió la morfometría, mismos que fueron correlacionados en los módulos RATIONAL y HECHMS (incluidos en el programa de cómputo Watershed Modeling System, WMS v. 8.1).
En la segunda etapa se estableció la precariedad urbana, a través de la aplicación del método de Análisis Multicriterio, la que que fue dividida por contornos urbanos para establecer las diferentes áreas de análisis. La última etapa corresponde a la correlación de las zonas de inundaciones con la precariedad para establecer las zonas de riesgo a inundaciones.

Entre los principales insumos, se contó con datos digitales como: modelo digital de elevación elaborado a partir de la modificación de las curvas de nivel de Corona (2009) mediante los vectores de la carta topográfica digital de Morelia con clave E14-A23. Así también se utilizaron programas de cómputo, destacando ArcGis, ArcView, WMS, MATLAB, Photoshop, CorelDraw y SPSS, con los cuales se realizaron los productos y bases cartográficas de la zona de estudio, así como la edición y aplicación de los métodos que a continuación se describirán. 
Identificación de áreas precarias a través de análisis multicriterio

La modelación de las áreas precarias se obtuvo a partir de la modificación del modelo diseñado por Vieyra y Larrazabal (2009), con lo cual la precariedad se estableció para el año 2005 utilizando variables similares acorde con la disponibilidad de información.

Para la producción del modelo se utilizó la información por AGEB (Área Geoestadística Básica ${ }^{5}$ del XII Censo de Población y Vivienda (INEGI, 2000), así como del II Conteo de Población y Vivienda 2005 (INEGI, 5 Unidad territorial de mayor detalle existente para el
manejo de las variables estadísticas analizadas.
2005), destacando la información espacial y categórica. Para su diseño se emplearon los software ArcView (versión 3.2), ArcGis (versión 9.0), ILWIS (versión 7.0) y SPSS (versión 17.0). Cabe mencionar que la información estadística disponible en los Censos y Conteos, así como las bases cartográficas de los AGEB difieren entre ellos, haciendo casi imposible una comparación, por lo que se tuvo que diseñar una base de datos al 2005 acorde a los objetivos de este trabajo, descartando aquellos indicadores que no presentaran relación con la precariedad

Con el propósito de reconocer las áreas precarias, las variables fueron clasificadas en tres categorías de análisis, la primera refiere a la calidad de las viviendas, la segunda a aspectos económicos y la tercera a la exclusión social (Cuadro $N^{0} 1$ ), estas a su vez proporcionaron las bases para establecer la preca-

\section{Cuadro $\mathrm{N}^{\circ} 1$}

Variables de caracterización del área de estudio

\begin{tabular}{|l|l|}
\hline \multicolumn{1}{|c|}{ Grupo temático } & \multicolumn{1}{|c|}{ Variable } \\
\hline Caracterización & $\begin{array}{l}\text { Población total } \\
\text { Densidad de población } \\
\text { Total de viviendas particulares habitadas }\end{array}$ \\
\hline \multirow{5}{*}{ Ingresos } & $\begin{array}{l}\text { Viviendas particulares habitadas con piso de tierra } \\
\text { Viviendas particulares habitadas con un solo cuarto } \\
\text { Viviendas particulares habitadas que no disponen de drenaje } \\
\text { Viviendas particulares que no disponen de alguno de los tres servicios } \\
\text { (agua potable, drenaje y electricidad) } \\
\text { Viviendas particulares habitadas sin ningún bien } \\
\text { Población derechohabiente a servicios de salud } \\
\text { Población sin derechohabiencia a servicios de salud } \\
\text { Relación de dependencia ajustada } \\
\text { Viviendas particulares habitadas que no disponen de lavadora } \\
\text { Viviendas particulares que no disponen de computadora } \\
\text { Población sin derechohabiencia a servicios de salud } \\
\text { Población de } 5 \text { años y más que habla alguna lengua indígena } \\
\text { Población de } 5 \text { años y más que habla alguna lengua indígena y no } \\
\text { habla español } \\
\text { Población de } 15 \text { años y más sin escolaridad } \\
\text { Población de } 15 \text { años y más analfabeta }\end{array}$ \\
\hline
\end{tabular}

Fuente: Elaboración propia. 
riedad urbana a través del método de análisis multicriterio.

En primera instancia para el desarrollo del método de análisis multicriterio, se determinó que las variables utilizadas fueran de tipo Ratio, lo que indica que los datos fueron categorizados en una escala donde el valor absoluto entre dos parámetros es independiente de la unidad de medida. Con lo cual, los indicadores se ponderaron según el nivel, esto es, la repartición empírica de un peso específico para cada uno de los indicadores en un rango de 0 a 100.

El siguiente paso en la construcción del método fue la estandarización, diseñada a partir de ecuaciones lineales que ajustaron los valores Ratio a una escala de comparación similar, así también, a las variables nominales o categóricas se les asignó un valor inicial numérico, mismo que posteriormente fue ajustado. En base a ello, la estandarización lineal resultó de la aplicación de la ecuación de una curva cóncava a través de un diagrama de frecuencia, donde la distribución de los datos se ajustó de mejor forma.

A partir de la ponderación de las variables y la aplicación del análisis multicriterio se obtuvieron cinco niveles de precariedad para la ciudad; muy bajo, bajo, medio, alto y muy alto. Estos niveles a su vez fueron delimitados por secciones, correspondientes a los contornos urbanos (contorno central, periferia inmediata y periferia exterior) diseñados por Vieyra y Larrazabal (2009), con los cuales se espacializó la información de precariedad y se definieron los asentamientos precarios de la periferia urbana de Morelia.

\section{Perímetro de inundación y análisis de} la variable de precipitación media

Con base en lo establecido en párrafos anteriores, la modelación de las zonas de inundaciones parte del análisis de la precipitación y su vinculación con parámetros morfométricos de la zona de estudio. Cabe destacar que dicho modelo fue precedido de elementos teóricos (Silva y Arreygue, 2005; Arreygue, 2007) así como de algunos modelos anteriormente diseñados correspondientes a la zona de estudio (Corona, 2009; Hernández, 2007). El interés por realizar el modelo se fundamentó en caracterizar la incidencia de inundaciones a través de rangos (muy bajo, bajo, medio, alto y muy alto), los que que serán correlacionados con los niveles de precariedad.

\section{Análisis espectral de la precipitación}

Dentro de los elementos que condicionan las inundaciones urbanas, la precipitación es de las variables principales en el análisis de la amenaza. Uno de los puntos importantes en cuanto a precipitación se refiere, es tratar de conocer su variación y frecuencia. Para ello se utilizó el método de Análisis Espectral, mediante los registros de precipitación media de la Comisión Nacional del Agua (CNA) de la estación Morelia, la cual cubre una serie de tiempo de 61 años (1947-2008).

\section{Método de análisis espectral de los procesos aleatorios}

Los procesos que se presentan en la atmósfera, generalmente se encuentran relacionados con procesos aleatorios de la variabilidad espacio-temporal de los fenómenos observados. De la misma forma, esta variabilidad de los procesos en la naturaleza se realiza no solo en el tiempo, sino también en coordenadas espaciales, por tal razón, los procesos geofísicos se relacionan con campos multidimensionales escalares o vectoriales.

El método empleado en este trabajo para encontrar las frecuencias más importantes es el Análisis Espectral; observando información oculta y discontinuidades en la serie de tiempo que no puede ser vista por medio de la estadística normal.

La transformada directa de Fourier es una ecuación para convertir datos de dominio del tiempo al dominio de frecuencias, y viceversa. En otras palabras, la transformada de Fourier identifica las diferentes frecuencias que componen una serie (senos y cosenos) con sus respectivas amplitudes y fases. Matemáticamente se expresa de la siguiente forma:

$$
\begin{array}{r}
S_{x}(\omega)=\int_{0}^{T} x(t) e^{-i 2 \pi \omega t} d t=\int_{0}^{T} x(t) \cos (2 \pi \omega t) \\
d t-i \int_{0}^{T} x(t) \operatorname{sen}(2 \pi \omega t) d t=a_{x}(\omega)-i b(\omega)
\end{array}
$$


Donde:

$x(t)$ : es la serie de tiempo;

$T$ : es la longitud total de la serie; y

$\omega$ : es la frecuencia. En donde la sección izquierda de la segunda igualdad es la parte real de la serie, mientras que la sección derecha es la parte imaginaria de la serie.

La función del periodograma físicamente otorga los periodos de una serie de tiempo, el autoperiodograma $S_{x x}(\omega)$

$$
S_{x x}(\omega)=\frac{1}{T} c_{x}(\omega) c_{x} *(\omega)
$$

Cuando el valor promedio del periodograma (según el conjunto de series) es igual a la densidad espectral, quiere decir que el periodograma es una estimación asintótica insesgada. De tal forma, la dispersión del periodograma es aproximadamente igual al cuadrado del valor promedio de la estimación, esto es, el cuadrado de la densidad espectral del mismo proceso, prácticamente, no depende de la longitud de la serie inicial. Por lo tanto, el periodograma, al mismo tiempo, es una estimación inconsistente del espectro.

\section{Modelación del perímetro de inundación}

El modelo para determinar las zonas de inundaciones se desarrolló a partir de diferentes fases, las cuales se fueron integrando en un Sistema de Información Geográfico (SIG). La primera fase correspondió a la elaboración de elementos geométricos, como curvas de nivel, cauces principales y secciones transversales. La segunda fase concernió a la obtención e integración de información sobre precipitación, escorrentía, volumen de agua, hipsometría, coeficientes de escurrimiento, longitud y superficie del área de estudio, mismos que fueron establecidos en los módulos RATIONAL y HEC-HMS dentro del WMS.

Cabe mencionar que al utilizar los módulos fue necesario establecer la capa de uso de suelo y los coeficientes de escurrimiento. Por lo tanto, los usos de suelo se digitalizaron a partir de la información de la Secretaría de Urbanismo y Medio Ambiente (SUMA), generalizando la clasificación en cinco usos; Agrí- cola, Bosque, Comercios y Servicios, Equipamiento, Habitacional mixto, Habitacional e Industrial. Por otra parte, los coeficiente de escurrimiento fueron utilizados de acuerdo al índices del Soil Conservation Service (SCS) que fueron modificados para la República Mexicana (Aparicio, 2005).

Con las bases establecidas, el último paso fue la integración de cada uno de los elementos para obtener la cartografía temática de las zonas de inundaciones y la precariedad urbana.

\section{Resultados}

De forma secuenciada se presentan a continuación los resultados obtenidos en cada uno de los métodos utilizados, partiendo de la caracterización de la precariedad y su interrelación con las zonas de inundación a partir del crecimiento urbano.

\section{El creciente suelo urbano \\ en la ciudad de Morelia}

La ciudad de Morelia desde su fundación ha pasado por diferentes etapas de urbanización, caracterizadas por la consolidación de la ciudad, el movimiento de independencia, conflictos internos de poder, la revolución mexicana, crisis políticas y recuperaciones económicas y demográficas (Vargas, 2008; Ávila, 2007). Sin embargo, a partir de la segunda mitad del siglo XX, la ciudad comenzó a experimentar un crecimiento urbano significativo e irreversible, el cual ha incidido en la reconfiguración territorial y en la ocupación periférica actual.

Durante la décadas de los treinta y los cuarenta la ciudad presentaba un crecimiento poblacional relativamente bajo $(40.000$ y 44.304 habitantes, respectivamente) y un proceso urbano sin grandes fluctuaciones. Así también, en los cuarenta se formaron las primeras colonias y se presentó una ligera expansión hacia los márgenes de los principales ríos, propiciando las bases en el incremento considerable de suelo urbano y poblacional, situación que se extendería durante las dos décadas siguientes.

Para el año de 1970 la población se incrementó a 161.040 habitantes (Figura $N^{\circ} 2$ ), 
este crecimiento demográfico exponencial influyó en gran medida en el aumento de asentamientos humanos sobre los límites de la ciudad (cubriendo una superficie de 3.500 hectáreas), ocupando suelos sin una vocación urbana y presentando problemas de inundaciones debido a los recurrentes desbordamientos del río Grande.

A partir del año de 1980 el proceso urbano se hizo más evidente, principalmente con los constantes desplazamientos hacia la periferia de la ciudad, ocupando suelos de difícil acceso para la dotación de servicios, marcados bajo su irregularidad y situados en zonas naturales de anegación. El número de colonias se incrementó a 65 en una superficie de 4.000 hectáreas y con una población de 297.544 habitantes (Consejo Nacional de Población, 2006). Para 1990 la ciudad estaba conformada por 256 colonias en una superficie de 5.427,3 hectáreas con un total de 480.269 habitantes (Tapia y Vargas, 2006), mientras que al año 2000 la ciudad presentaba un total de 549.996 habitantes, mientras que el número de colonias se incrementó a 350. Ya para el año 2005 la ciudad estaba conformada por más de 600 colonias con una población de 608.049 habitantes en una superficie de 9.804,4 hectáreas. Estos factores ponen de manifiesto que el proceso de urbanización de los últimos treinta años ha representado una nueva dinámica en el crecimiento de la ciudad, destacando el muy corto tiempo en el que este se ha presentado.

La fuerte dinámica demográfica y la presión que esta ha generado en la ocupación de nuevos espacios en la periferia de la ciudad, principalmente a partir de la década de los ochenta, reflejan las debilidades de los planes de desarrollo urbano que no han sido capaces de frenar la expansión de los límites de la ciudad, situación que, acorde a las estimaciones demográficas, continuará durante los próximos 20 años (Cuadro № 2). De la misma forma, los intentos por disminuir y ralentizar el excesivo crecimiento de superficie urbana han fracasado; por otra parte se destaca el notorio cambio en el uso del suelo de agrícola a urbano durante los últimos 35 años (López et al., 1999), destacando sitios donde su giro cambió radicalmente al uso habitacional y habitacional mixto $(65 \%)$, en los cuales comúnmente se edifican viviendas autoconstruidas sujetas a condiciones precarias que poco a poco se van consolidando.

Figura $\mathrm{N}^{\circ} 2$

Crecimiento demográfico en la ciudad de Morelia

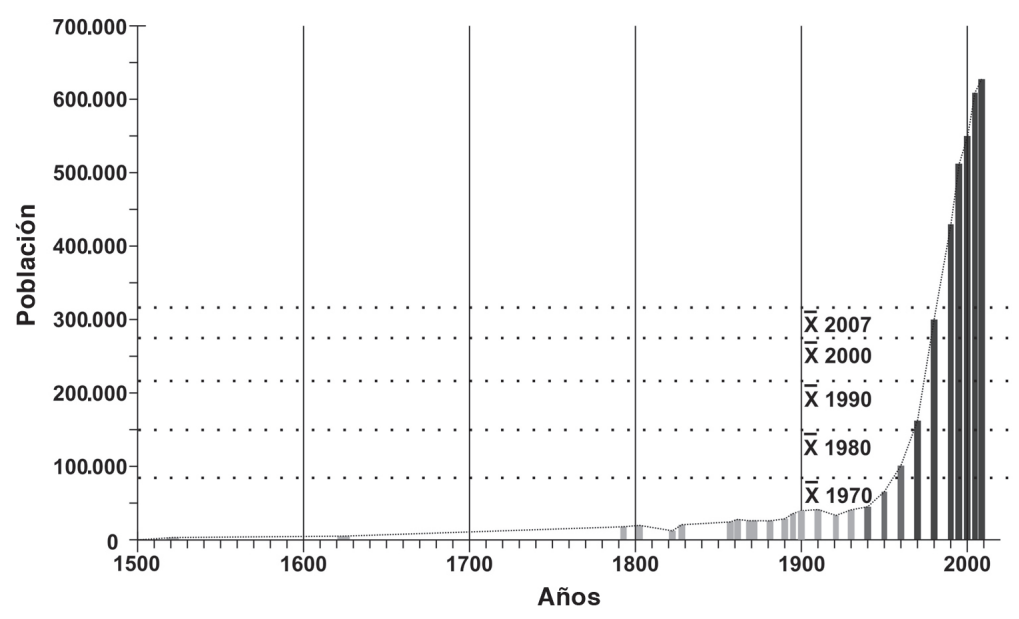

Fuente: Elaboración propia en base a Vargas, 2008. 
Cuadro $\mathrm{N}^{\circ} 2$

Estimaciones del crecimiento poblacional (1990-2030)

\begin{tabular}{|c|c|c|c|c|}
\hline \multirow{2}{*}{ Año } & Población total & \multicolumn{3}{|c|}{ Población estimada } \\
\cline { 3 - 5 } & & Método Aritmético* & Método Geométrico* & Promedio \\
\hline 1990 & 428.486 & - & - & - \\
2000 & 549.996 & - & - & - \\
2005 & 608.049 & - & - & - \\
2010 & - & 686.183 & 682.932 & 684.557 \\
2015 & - & 774.357 & 767.037 & 770.697 \\
2020 & - & 873.862 & 861.500 & 867.681 \\
2030 & - & 1.098 .444 & 1.086 .760 & 1.092 .602 \\
\hline
\end{tabular}

* Las tasas de crecimiento inicial para el método aritmético fue de 2,57, mientras que para el método geométrico fue de 2,35.

Fuente: Elaboración propia en base a la información censal de INEGI para 1990, 2000 y el conteo de 2005.

Estos parámetros indican las facilidades para la urbanización sobre la periferia, donde la invasión, procesos de corruptela, especulación o malas decisiones han aumentado los enclaves precarios sobre suelos en riesgo de inundaciones. Así también, las condiciones de la población están sujetas a las carencias de alimentación, empleo, seguridad social y educación, donde su principal resguardo son las viviendas autoconstruidas de materiales deficientes, carentes de servicios e irregularidad en la tenencia de la tierra.

\section{Contornos urbanos}

Con base en lo establecido en el apartado anterior, se determinó utilizar y modificar como sectores de análisis los tres contornos urbanos diseñados por Vieyra y Larrazabal (2009), los cuales se relacionan con los procesos urbanos descritos en este trabajo. El primer contorno denominado central está conformado por el crecimiento urbano alcanzado hasta los años setenta. El segundo contorno corresponde a la primera fase importante de expansión periférica de la ciudad, que sostuvo hasta el año noventa y se le denomina periferia inmediata. $Y$ el tercer contorno está reconocido principalmente por los asentamientos periféricos actuales, denominado periferia exterior y que se encuentran bajo un proceso de urbanización.
Cabe destacar que los tres contornos presentan una marcada tendencia por el uso de suelo habitacional y habitacional mixto, sin embargo, en la periferia exterior a raíz de la consolidación de población mayoritariamente de bajos recursos y la presión que sostiene, supone que los niveles de precariedad pudieran ser más altos que en los otros dos contornos (Figura $\mathrm{N}^{\circ} 3$ ).

\section{Precariedad en la periferia de la ciudad de Morelia}

En relación a los AGEB del año 2000 y 2005, el contorno de la periferia inmediata es el que presenta la mayor superficie y población de la ciudad, sin embargo, el contorno central presenta casi la misma población en la mitad de superficie de la periferia inmediata (Cuadro $\mathrm{N}^{\circ} 3$ ). A pesar de estos factores el contorno central no ha presentado grandes cambios, por el contrario, disminuyó su población. Por su parte, el contorno de la periferia inmediata presentó un ligero crecimiento, pero los valores de población se mantuvieron cercanos al $50 \%$ del total de la ciudad.

Por otro lado, el proceso que presenta la periferia exterior en tan solo cinco años, destaca sobre los otros dos contornos, con un aumento de la población de 36.597 habitan- 
Figura $\mathrm{N}^{\circ} 3$

Principales usos de suelo en la ciudad de Morelia, 2008

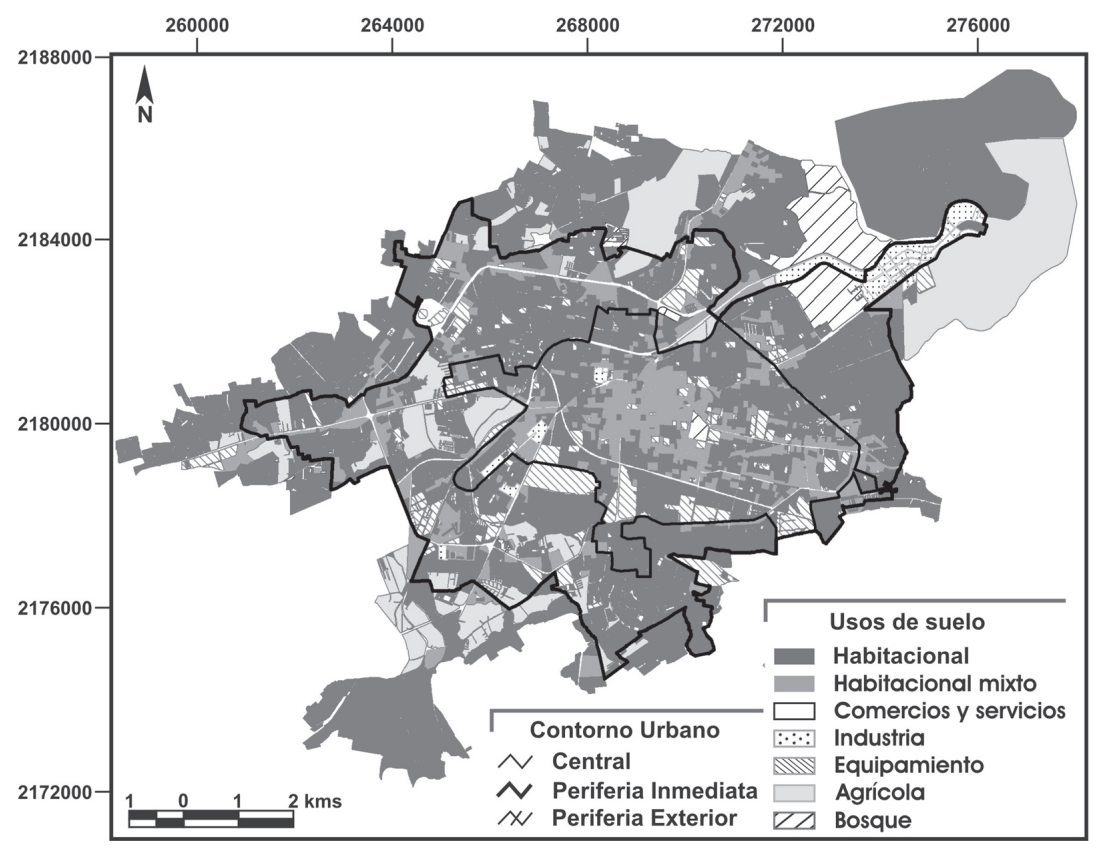

Fuente: Elaboración propia en base a la Secretaría de Urbanismo y Medio Ambiente.

Cuadro $\mathrm{N}^{\circ} 3$

Tabla general de los AGEB para los años 2000 y 2005

\begin{tabular}{|l|c|r|r|r|r|}
\hline \multicolumn{7}{|c|}{ Sección urbana / Año } & Área (ha) & AGEB & Área (\%) & Población total & Población (\%) \\
\hline \multicolumn{7}{|c|}{2000} \\
\hline Centro & $2.340,3$ & 58 & 26 & 242.855 & 44 \\
Periferia inmediata & $4.685,4$ & 111 & 51 & 269.449 & 49 \\
Periferia exterior & $2.112,5$ & 51 & 23 & 37.692 & 7 \\
Total & $9.138,2$ & 220 & 100 & 549.996 & 100 \\
\hline \multicolumn{7}{|c|}{2005} \\
Centro & $2.340,3$ & 58 & 24 & 247.015 & 41 \\
Periferia inmediata & $4.750,2$ & 117 & 48 & 286.745 & 47 \\
Periferia exterior & $2.713,9$ & 90 & 28 & 74.289 & 12 \\
Total & $9.804,4$ & 266 & 100 & 608.049 & 100 \\
\hline
\end{tabular}

Fuente: Elaboración propia. 
tes y un incremento de la superficie de 601,4 hectáreas. Estos factores se relacionan a la fuerte presión de consumo del suelo a la que ha sido sometido el contorno, principalmente por la demanda constante de suelo para viviendas por parte de población de bajos recursos, mismos que han encontrado en este contorno suelos a bajo costo de fácil adquisición, cercanos al contorno central y caracterizados por sus condiciones de precariedad.

De la misma forma, en el Cuadro $\mathrm{N}^{\circ} 4$ se puede observar como el nivel medio de precariedad en la ciudad no representa lo que sucede en cada uno de los contornos. Por lo tanto, se demuestra que el contorno central está compuesto por una precariedad baja, estimulado por la función de centro de comercio, equipamiento y habitacional, así también sus áreas de amortiguamiento se encuentran bordeadas por habitantes de clase media a clase alta.

En lo referente a la periferia inmediata, las viviendas autoconstruidas, la disposición de servicios, ingresos y derechohabiencia al sector salud caracterizan sus condiciones de precariedad. Este contorno presenta niveles que van del rango muy bajo al alto, siendo el nivel medio el que presenta la mayor población con 164.088 habitantes en una superficie del $57 \%$ del contorno. Por su parte, el rango bajo está representado por 98.568 habitantes en un área de 1.592,6 hectáreas. Sin embargo, cabe destacar la precariedad alta, representada en el $7 \%$ de la superficie con una población de 19.514 habitantes, situación que influye en los factores de susceptibilidad ante las amenazas naturales.

La periferia exterior representa la mayor estratificación de rangos, los cuales van de baja a muy alta precariedad. En esta sección se destaca la precariedad alta, representada por el $43 \%$ de la superficie y una población de 31.983 habitantes, mientras que la precariedad muy alta se localiza en el $15 \%$ de la superficie con una población de 11.144 habitantes. Entre estos dos rangos de precariedad suman el $58 \%$ de la superficie y una población de 43.127 habitantes, lo que equivale a que la mayor parte del contorno se encuentra en condiciones de precariedad, representados por viviendas autoconstruidas con materiales naturales y ligeros (madera, cartón y plásti$\mathrm{co})$, inseguridad en la tenencia de la tierra (suelo ejidal o comunal) y carencias de los servicios básicos (electricidad, agua y drenaje). De este último factor se destaca el acceso al agua potable, donde el número de vivien-

Cuadro $\mathrm{N}^{\circ} 4$

Nivel de precariedad por sección urbana, 2005

\begin{tabular}{|l|l|c|c|c|c|c|c|}
\hline Contornos & Variables & Muy alta & Alta & Media & Baja & Muy baja & Total \\
\hline Centro & Área (ha) & - & - & 194,4 & 1815,7 & 330,3 & 2340,4 \\
& Área (\%) & - & - & 8 & 78 & 14 & 100 \\
& $\begin{array}{l}\text { Población } \\
\text { promedio }\end{array}$ & - & - & 20.515 & 191.642 & 34.858 & 247.015 \\
Periferia & Área (ha) & - & 315,3 & 2651,3 & 1592,6 & 73,9 & 4633,1 \\
inmediata & Área (\%) & - & 7 & 57 & 34 & 2 & 100 \\
& $\begin{array}{l}\text { Población } \\
\text { promedio }\end{array}$ & & 19.514 & 164.088 & 98.568 & 4.576 & 286.745 \\
$\begin{array}{l}\text { Periferia } \\
\text { exterior }\end{array}$ & $\begin{array}{l}\text { Área (ha) } \\
\text { Área (\%) }\end{array}$ & 424,7 & 1218,8 & 948,7 & - & 238,8 & 2830,9 \\
& $\begin{array}{l}\text { Población } \\
\text { promedio }\end{array}$ & 11.144 & 31.983 & 24.896 & - & 6.266 & 74.289 \\
\end{tabular}

Fuente: Elaboración propia. 
das carentes de este servicio, se incrementó a casi el doble en tan solo 5 años, lo que equivale al $10 \%$ de las viviendas particulares en la ciudad (Cuadro No 5 ).

La Figura $N^{\circ} 4$ muestra el mapa síntesis de los niveles de precariedad acorde a los AGEB para cada uno de los contornos, donde se observa que las periferias (inmediata y exterior) presentan una estratificación de la precariedad mayor que la central, en este sentido y de acuerdo a los planteamientos del trabajo, se reconoce que la periferia exterior presenta los mayores problemas de precariedad, principalmente al poniente, sur y norte de la ciudad, siendo este último sector el que muestra los niveles más altos de precariedad, representado por 196 hectáreas y una población de 134.887 habitantes.

Entre las colonias que conforman la periferia exterior con un nivel de precariedad muy alta se encuentran las de reciente aparición (finales de la década de los noventa), tales como Gertrudis Sánchez, Medallistas Olímpicos, Pastor Sánchez y Valle del Real. Cabe mencionar que estas colonias han sido afectadas severamente por las inundaciones de los últimos años, que acorde a sus condiciones precarias han experimentado daños en la vivienda, o en casos extremos la pérdida parcial o total de la misma.

\section{Inundaciones y precariedad en la periferia urbana}

La fuerte dinámica que presenta la periferia de la ciudad de Morelia, no solo se refleja en la fragilidad de las comunidades precarias, sino en la recurrencia de eventos de inundaciones que en los últimos años se han presentado. Estos eventos se destacan entre muchos otros factores por fuertes precipitaciones de gran intensidad y magnitud, que en relación a los procesos urbanos pudieran engrandecer la problemática de las inundaciones. Por ello, en la determinación de las zonas de inundaciones para la ciudad, es indispensable realizar el análisis de la precipitación en los últimos cincuenta años, con el objeto de encontrar discontinuidades que proporcionen información adicional en la formación de las inundaciones (Figura $N^{\circ} 5$ ).

A través de la Figura $N^{0} 5$ se observan los registros de la precipitación media para la serie de tiempo de 1947 al 2008, la cual se dividió en dos periodos principales, mismos que fueron correlacionados con los procesos urbanos posteriores a la segunda mitad del siglo XX. En relación al primer periodo, se observan eventos que presentaron registros superiores a los $200 \mathrm{~mm}$, a su vez, la información de inundaciones para este periodo destacan los constantes desbordamientos del

Cuadro $\mathrm{N}^{\circ} 5$

Vivienda sin acceso a agua y drenaje

\begin{tabular}{|c|c|c|c|}
\hline Variables & Centro & $\begin{array}{c}\text { Periferia } \\
\text { inmediata }\end{array}$ & $\begin{array}{l}\text { Periferia } \\
\text { exterior }\end{array}$ \\
\hline \multicolumn{4}{|l|}{2000} \\
\hline Total de viviendas particulares habitadas & 56.812 & 60.088 & 8.485 \\
\hline Viviendas particulares habitadas que no disponen de agua & 53.785 & 52.737 & 3.839 \\
\hline Viviendas particulares habitadas que no disponen de drenaje & 134 & 1.053 & 1.779 \\
\hline \multicolumn{4}{|l|}{2005} \\
\hline Total de viviendas particulares habitadas & 56.815 & 74.468 & 14.651 \\
\hline Viviendas particulares habitadas que no disponen de agua & 53.790 & 66.937 & 6.123 \\
\hline Viviendas particulares habitadas que no disponen de drenaje & 86 & 480 & 3.701 \\
\hline Total de viviendas 2005 & 53.876 & 67.417 & - \\
\hline
\end{tabular}

Fuente: Elaboración propia. 
Figura $\mathrm{N}^{\circ} 4$

Precariedad urbana en la ciudad de Morelia, 2005

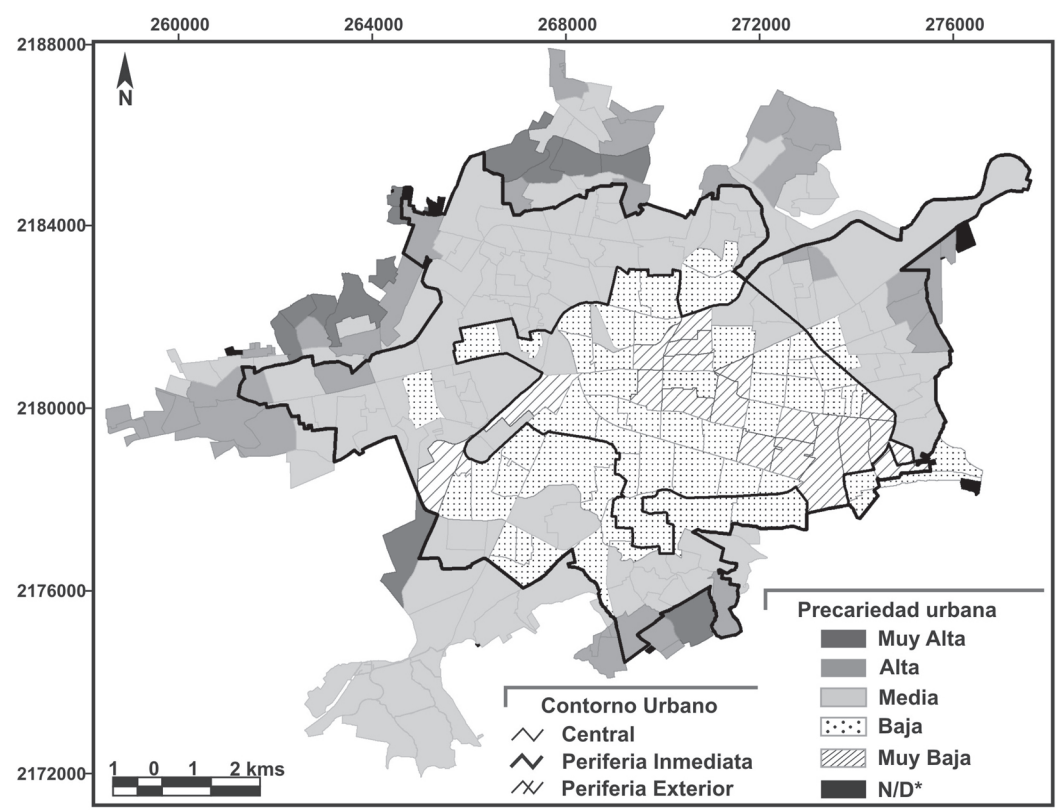

* Sin dato. Fuente: Elaboración propia.

Figura $\mathrm{N}^{\circ} 5$

Serie de tiempo de la precipitación media de la estación Morelia (1947-2008)
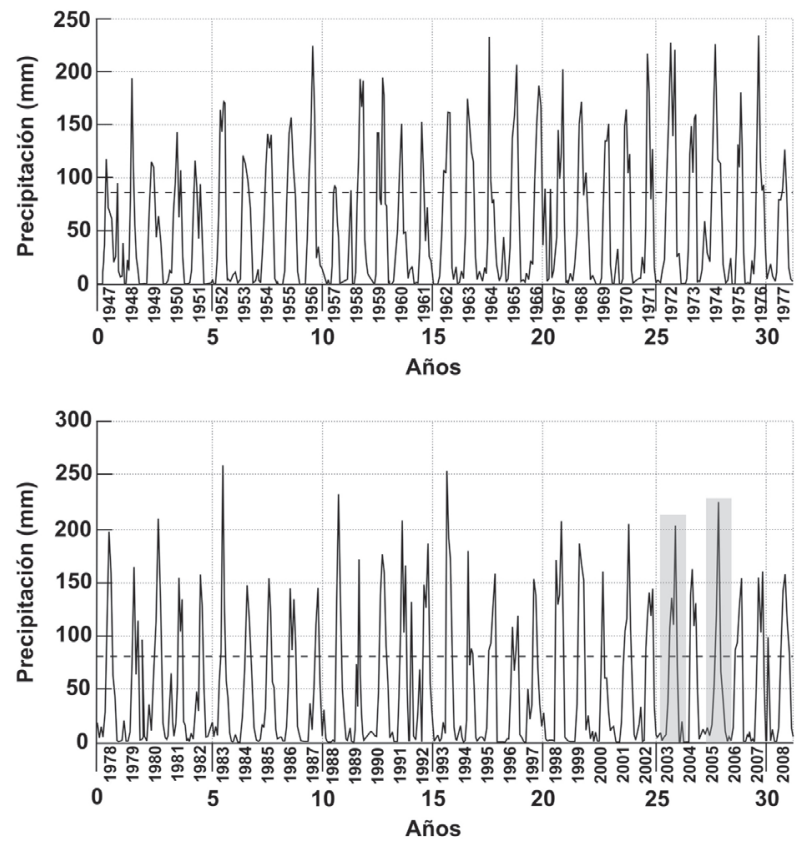

Fuente: Elaboración propia. 
río Chiquito, afectando considerablemente a la población del sector sur del contorno central y de la periferia inmediata.

En el segundo periodo también se observa una ciclicidad similar al anterior, con presencia de eventos que alcanzaron los 250 mm en los años de 1983 y 1993. De acuerdo a los antecedentes, este periodo se destaca por la presencia de inundaciones históricas que afectaron casi toda la ciudad, en especial las acontecidas en los años de 1998, 2003 y 2005 donde las fuertes precipitaciones oscilaron entre los $150 \mathrm{~mm}$ y $200 \mathrm{~mm}$, siendo los habitantes de la periferia inmediata y exterior los más afectados.

Ante tales circunstancias, actualmente $150 \mathrm{~mm}$ de lluvia propicia inundaciones en diversos sectores de la ciudad, situación que no ocurría en el primer periodo, donde se presentaron eventos que sobrepasaron los $250 \mathrm{~mm}$ sin las consecuencias desastrosas de los últimos años. Por lo tanto, los eventos de precipitación demostraron un patrón homogéneo en los sesenta años de registros, mientras que las inundaciones se asocian al aumento del área urbana y ocupación de zonas de riesgo, especialmente sobre el periurbano de la ciudad; parámetro que se corroborará con el modelo de inundaciones.

En la Figura $N^{\circ} 6$ se observa el área de inundaciones, la cual se presenta de forma transversal a la ciudad, delimitada por los cauces del río Grande y río Chiquito, así también, se aprecian puntos críticos al norte, centro, poniente y sur oriente de la ciudad, originados por la confluencia de escurrimientos (nodos de concentración). Esta situación demarca una población que constantemente está siendo afectada por eventos de inundaciones, principalmente en la periferia exterior, donde se localiza la población con los niveles más altos de precariedad.

De la misma forma, se puede observar que el sector norte (precariedad muy alta)

Figura $\mathrm{N}^{\circ} 6$

Correlación de los modelos de inundación y precariedad urbana

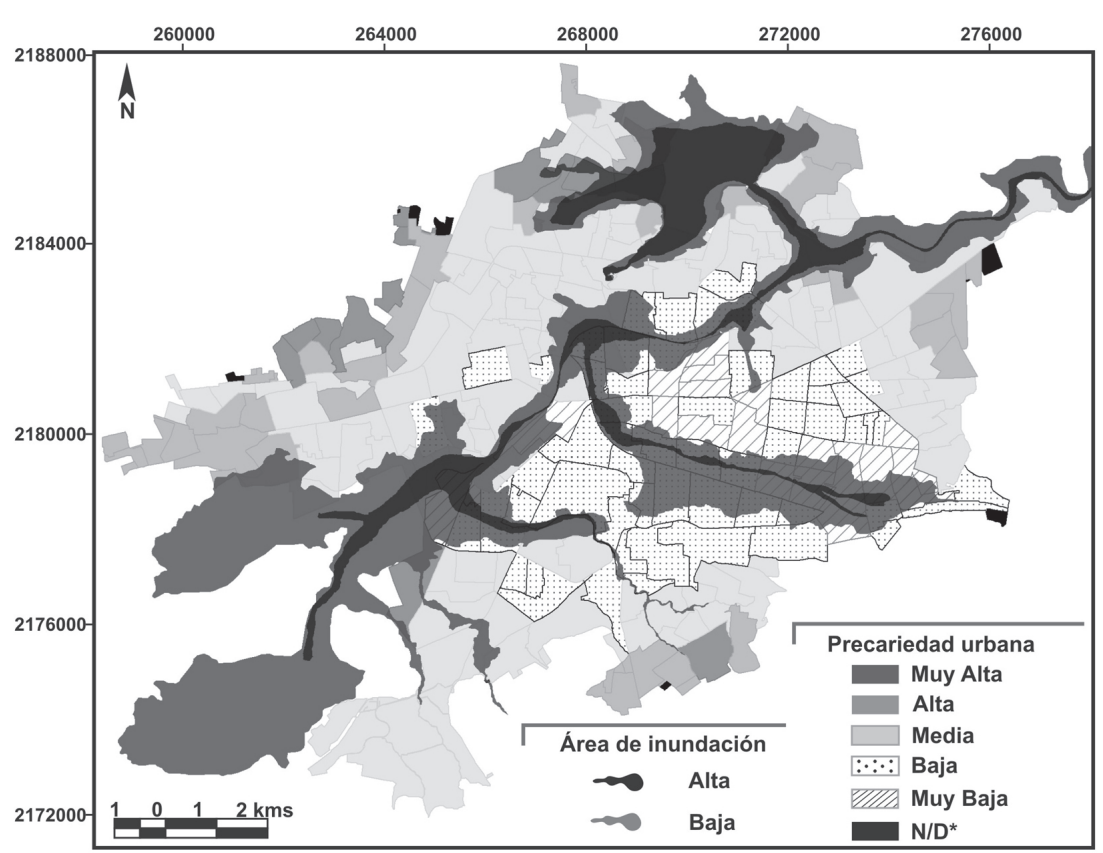

* No hay dato.

Fuente: Elaboración propia. 
descrito en el apartado anterior, se localiza sobre una zona baja, demarcada por una cuenca exorreica que deposita sus aguas sobre el río Grande. La dinámica que presenta el periurbano reflejan la deficiente planeación urbana, caracterizada por las irregularidades en la gestión urbana, así como la ignorancia, ocupación, corrupción, especulación e invasión de suelos no aptos para el uso urbano, a pesar de ello, la presión poblacional es marcadamente superior que el contorno central.

Los contornos urbanos presentes en el Cuadro $N^{\circ} 6$ muestran que 190.776 habitantes se encuentran en riesgo de inundaciones, mientras que la población no inundable equivale al $69 \%$, esto refiere que casi un $30 \%$ de la población de la ciudad de Morelia muestra problemas de inundaciones. Así también, casi la mitad de la población de la periferia exterior se encuentra en constante riesgo de inundaciones, condición que se agrava por las condiciones de alta y muy alta precariedad.

La población del contorno central representa el nivel más alto de población afectada por inundaciones, sin embargo, debido a los niveles bajos de precariedad que le caracterizan favorecen una mejor respuesta al impacto. Por el contrario, la periferia inmediata y periferia exterior suman casi la población del contorno urbano central en situación de riesgo, los cuales de acuerdo a sus condiciones precarias las hacen más susceptibles a las inundaciones, manifestándose en costosas y lentas recuperaciones.

Con base en los resultados de la correlación de los modelos, la población en el contorno urbano de la periferia exterior es la que presenta los mayores problemas, en especial la sección norte, caracterizada por los niveles más altos de precariedad localizados en zonas de riesgo de inundaciones. Las colonias que se destacan son Valle del Real, Gertrudis Sánchez, Pastor Ortiz, Solidaridad, El Lago 1, Valle de los Manantiales, MedaIlistas Olímpicos, Torreón Nuevo y Presa los Reyes. Entre las condiciones que caracterizan la precariedad de estas zonas se encuentra la mala materialidad de las viviendas, la falta de agua potable, electricidad, drenaje, infraestructura hidráulica, empleo, educación, derechohabiencia a servicios de salud y seguridad pública (Figura $\mathrm{N}^{\circ} 7$ ).

De la misma forma, las fuertes precipitaciones han sido uno de los detonantes en la formación de inundaciones, pero no es la principal, ya que los resultados de este trabajo demuestran que la falta de planeación y gestión urbana, así como la dinámica, presión y patrones de consolidación urbana que se han estado llevando a cabo en la zona norte de la periferia exterior, permiten vislumbrar que la situación no es nada alentadora para los próximos años, ya que se prevé que el número de personas en riesgo de desastre por inundaciones se incremente para los próximos años.

Cuadro $\mathrm{N}^{\circ} 6$

Población afectada por inundaciones según sección urbana, 2005

\begin{tabular}{|l|c|c|c|}
\hline \multicolumn{1}{|c|}{ Sección } & Población & Población afectada* & Población afectada (\%) \\
\hline Centro & 247.015 & 114.629 & 19 \\
Periferia inmediata & 286.745 & 54.222 & 9 \\
Periferia exterior & 74.289 & 21.925 & 4 \\
No inundable & - & 417.273 & 69 \\
Total & 608.049 & 190.776 & 100 \\
\hline
\end{tabular}

*Nota: Se interpoló la información de población, superficie y perímetro inundable en aquellas zonas donde el perímetro no cubría totalmente al AGEB.

Fuente: Elaboración propia. 
Figura $\mathrm{N}^{\circ} 7$

Asentamientos en la periferia de Morelia. Drenajes expuestos y precariedad de las viviendas

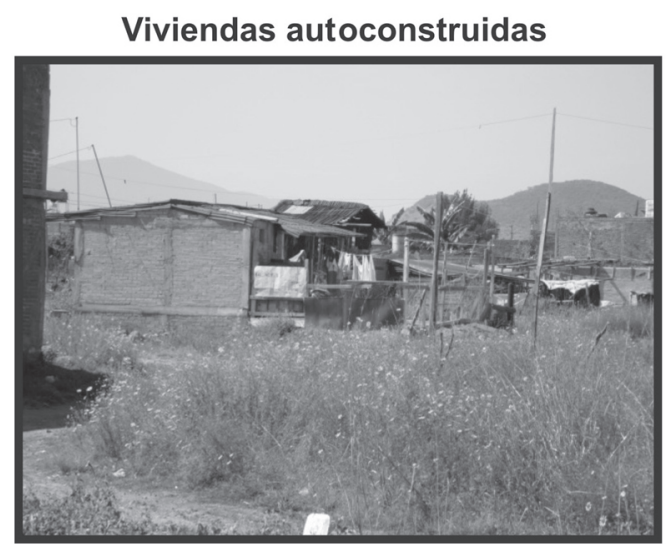

Deficientes materiales de construcción

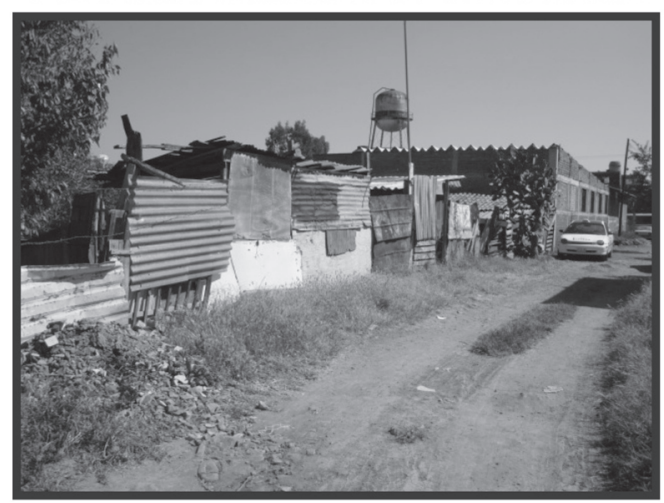

Fuente: Colección personal de los autores.

\section{Consideraciones finales}

Los resultados de este trabajo demuestran el rápido incremento de asentamientos periféricos en riesgo de inundaciones, sobre espacios que regularmente se encuentran fuera de la normatividad oficial y ocupados por población de bajos recursos económicos en búsqueda de vivienda. Esto a su vez se traduce en las condiciones de dichas viviendas, autoconstruidas con materiales precarios, carentes de los servicios básicos e irregularidad en la tenencia del suelo, fomentando la susceptibilidad y el riesgo.

La dinámica de crecimiento demográfico de la ciudad se encuentra representada por los escenarios de la periferia inmediata y ex-
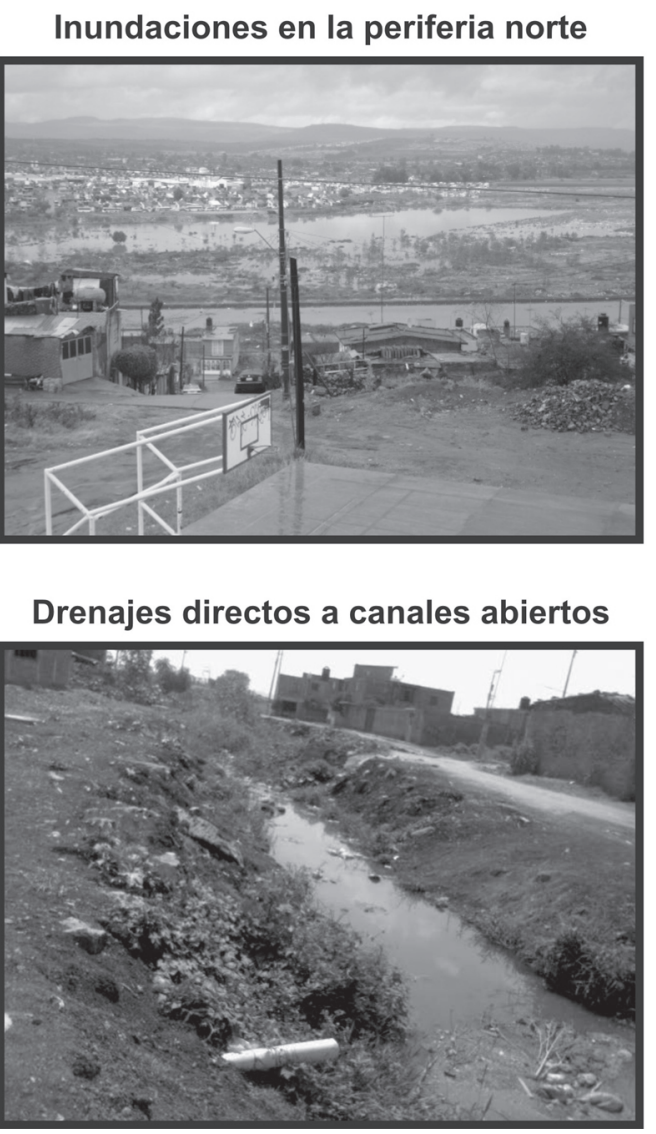

terior, debido al decremento poblacional que ha manifestado el contorno central desde los primeros años del 2000. A su vez la periferia inmediata concentra la mayor población y superficie urbana de la ciudad, sin embargo, la periferia exterior actualmente presenta ritmos superiores a los otros dos contornos, caracterizado por su alta precariedad e incidencia de inundaciones, mismos que se encuentran sujetos a la carencia del servicio de electricidad, agua potable, drenaje e infraestructura hidráulica, así como empleo, servicios de salud, educación y seguridad.

Las colonias sobre la periferia exterior con alta y muy alta precariedad en riesgo de inundaciones son Medallistas Olímpicos, Gertrudis Sánchez, Pastor Ortiz, Solidaridad, 
Valle del Real y Valle de los Manantiales, las cuales han ocupado estos espacios a través casos de negligencia, corruptela, intereses personales, ignorancia y necesidad. Así también, de no implementarse políticas públicas eficientes en el control del crecimiento urbano y en la mejora de las condiciones de vida de dichos sectores de la ciudad, sus habitantes seguirán quedando a merced del desastre.

Los registros de precipitación mantienen cierta homogeneidad en la serie de tiempo estudiada, manifestando que es un elemento fundamental en la formación de inundaciones pero no es la principal causa del desastre. De tal forma, uno de los factores principales que incrementan la formación del riesgo es el constante crecimiento del área urbana, el cual viene precedido del incremento demográfico, las condiciones de precariedad de la población, el incumplimiento de reglamentos y la carencia de una planeación adecuada que evite los asentamientos sobre zonas no aptas para uso urbano.

El desastre corresponde a procesos multifactoriales y multicausales, mismos que evolucionan a partir de condiciones críticas preexistentes que se transforman en situaciones desastrosas para ciertos grupos cuando ocurre el impacto de una amenaza natural (García, 2008). Este trabajo pone de manifiesto que el desastre se hace debido a que la recurrencia de inundaciones viene a la par del crecimiento urbano en superficies no aptas, representado por diversos asentamientos bajo condiciones precarias y ocupando suelos inseguros que suelen transformar el fenómeno natural en desastre.

\section{Agradecimientos}

Los autores agradecen la colaboración de Alejandra Patricia Larrazabal de la Vía por su apoyo en la conformación y manejo de bases de datos y resultados cartográficos.

\section{Referencias bibliográficas}

AGUILAR, A. G., \& VIEYRA, A. Urbanization, migrations, and employment in Latin America. A review of trends. In: JACKIEWICZ, E. y BOSCO, F. (edits.) Placing Latin America. Contemporary themes in human geography. Maryland: Rowman \& Littlefield Publishers, Inc., 2008, p. 51-68.
AGUILAR, A. G., y ESCAMILLA, I. (coord.) Periferia urbana: deterioro ambiental y reestructuración metropolitana. Ciudad de México: Editorial Miguel Ángel Porrúa, 2009.

APARICIO, M. F. Fundamentos de hidrología de superficie. Ciudad de México: Editorial LIMUSA, 2005.

ARREYGUE, R. E.; ALCALÁ, O. S. y SILVA, M. C. Cambios físicos importantes en los flujos del río Chiquito, Morelia, Michoacán. GEOS, 2005, № 1, p. 98-111.

ARREYGUE, R. E. Evaluación de las constantes inundaciones en la Ciudad de Morelia, Michoacán, México. En: Cuzco, $8^{\circ}$ Congreso Iberoamericano de Ingeniería Mecánica, 2007.

ÁVILA, G. P. Agua, ciudad y medio ambiente: una visión histórica de Morelia. Morelia: Universidad Nacional Autónoma de México, Secretaría de Desarrollo Social y $\mathrm{H}$. Ayuntamiento de Morelia/Observatorio urbano de Morelia, 2007.

AZÓCAR， G.; HENRÍQUEZ, C.; VALENZUELA, C. y ROMERO, H. Tendencias sociodemográficas y segregación socioespacial en Los Ángeles, Chile. Revista de Geografía Norte Grande, 2008, No 41, p. 103-128.

BULL-KAMANGA，L.; DIAGNE, K.; LAVELL, A.; LEON, M.; LERISE, F.; MACGREGOR, H.; MASKREY, A.; MESHACK, M.; PELLING, M.; REID, H.; SATTERTHWAITE, D.; SONGSORE, J.; WESTGATE, K. \& YITAMBE, A. From everyday hazards to disasters: the accumulation of risk in urban areas. Environment \& Urbanization, 2003, No 1, p. 93-204.

CANNON, T.; TWIGG, J. \& ROWELL, J. Social vulnerability, sustainable livelihoods and disaster. London: Conflict and humanitarian assistance department and sustainable livelihoods support office, 2004.

CARDONA, A. O. La necesidad de repensar de manera holística los conceptos de vulnerabilidad y riesgo. Una crítica y una revisión necesaria para la gestión. Panamá: Red de estudios sociales en prevención de desastres en América Latina, 
2003. Disponible en Internet: http://www. desenredando.org/public/articulos/2003/ rmhcvr_may-08-2003.pdf

CONSEJO NACIONAL DE POBLACIÓN. La situación demográfica de México 2006. Ciudad de México: Fondo Nacional de Población (UNFPA), 2006.

CORONA, N. Vulnerabilidad de la ciudad de Morelia a inundaciones. Tesis de Maestría en Geografía. Morelia: Centro de Investigaciones en Geografía Ambiental, Universidad Nacional Autónoma de México, México, 2009.

CORTÉS, F.; HERNÁNDEZ, D.; HERNÁNDEZ, L. E.; SZÉKELY, M. y VERA, L. $H$. Evolución y características de la pobreza en México en la última década del siglo XX. Nueva Época, 2003, No 2, p. 295-325.

CUTTER, S.; BORUFF, B. \& SHIRLEY, L. Social vulnerability to environmental hazards. Social Science Quarterly, 2003, No 2, p. 242-261.

GARCÍA, A. V. Estrategias adaptativas y amenazas climáticas. En: Urbina, J. y Martínez, J. (comps.). Más allá del cambio climático. Las dimensiones psicosociales del cambio ambiental global. Ciudad de México: Facultad de psicología UNAM, 2008, p. 1-17.

HERNÁNDEZ, V. Modelo de inundación Morelia. Modelo tomado por la Secretaría de Urbanismo y Medio Ambiente. Morelia: Departamento de geología y mineralogía, Universidad Michoacana de San Nicolás de Hidalgo, 2007. Disponible en Internet: http:// suma.michoacan.gob.mx/pdf/OET_Doga/ Atlas/morelia_web.swf

INSTITUTO NACIONAL DE ESTADÍSTICA GEOGRAFÍA E INFORMÁTICA (INEGI). XII Censo general de población y vivienda. CINCE. Información digital. Aguascalientes: Instituto Nacional de Estadística Geografía e Informática, 2000.

INSTITUTO NACIONAL DE ESTADÍSTICA GEOGRAFÍA E INFORMÁTICA (INEGI). II Conteo de población y vivienda. Información digital. Aguascalientes: Instituto Nacional de Estadística Geografía e Informática, 2005.
LAVELL, A. Riesgo, desastre y territorio. La necesidad de los enfoques regionales/ transnacionales. Anuario Social y Político de América Latina y el Caribe. Nueva Sociedad, 2002, No 5, p. 140-147.

LÓPEZ, G. E. Cambio de uso de suelo y crecimiento urbano en la ciudad de Morelia. Tesis de Maestría. Morelia: División de Ciencias y Humanidades, Facultad de Biología, Universidad Michoacana de San Nicolás de Hidalgo, México, 1999.

PELLING, M. Measuring vulnerability to urban disaster risk. Open House International on managing urban disasters, 2005, $\mathrm{N}^{0} 1, \mathrm{p}$. 125-132.

SECRETARÍA DE DESARROLLO SOCIAL. Delimitación de las zonas metropolitanas de México 2005. Ciudad de México: Secretaría de Gobernación de México en colaboración con el Instituto Nacional de Estadísticas, Geografía e Informática y el Consejo Nacional de Población, 2007.

SILVA, M. y ARREYGUE, R. Estudio preliminar de los peligros hidrológicos e hidráulicos de la ciudad de Morelia, Michoacán. GEOS, 2005, № 1, p. 112-125.

TAPIA, C. y VARGAS, G. El impacto del desarrollo urbano en los recursos naturales. Cuadernos de Investigación y Difusión, 2006, vol. I, p. 69-153.

VARGAS, G. Urbanización y configuración territorial en la región de Valladolid-Morelia 1541-1991. Morelia: Morevallado Editores, 2008.

VIEYRA, A. y LARRAZABAL, A. Urbanización y precariedad urbana en la ciudad de Morelia, Michoacán, México. En: Montevideo, XII Encuentro de Geógrafos de América Latina, 2009.

ZULAICA, L. y CELEMÍN, J. Análisis territorial de las condiciones de habitabilidad en el periurbano de la ciudad de Mar del Plata (Argentina), a partir de la construcción de un índice y de la aplicación de métodos de asociación espacial. Revista de Geografía Norte Grande, 2008, № 41, p. 129-146. 